\title{
Effect of untreated bed nets on blood-fed Phlebotomus argentipes in kala-azar endemic foci in Nepal and India
}

\author{
Albert Picado ${ }^{1 /}$, Vijay Kumar ${ }^{4}$, Murari Das², Ian Burniston', Lalita Roy², Rijal Suman², \\ Diwakar Dinesh ${ }^{4}$, Marc Coosemans ${ }^{3}$, Shyam Sundar ${ }^{5}$, Kesari Shreekant ${ }^{4}$, Marleen Boelaert ${ }^{3}$, \\ Clive Davies ${ }^{1}$, Mary Cameron ${ }^{1}$
}

${ }^{1}$ London School of Hygiene and Tropical Medicine, Keppel Street, London WC1E 7HT, UK ${ }^{2}$ BP Koirala Institute of Health Sciences, Dharan, Nepal ${ }^{3}$ Institute of Tropical Medicine, Antwerp, Belgium ${ }^{4}$ Rajendra Memorial Research Institute of Medical Sciences, Patna, India ${ }^{5}$ Banaras Hindu University, Varanasi, India

Observational studies in the Indian subcontinent have shown that untreated nets may be protective against visceral leishmaniasis (VL). In this study, we evaluated the effect of untreated nets on the blood feeding rates of Phlebotomus argentipes as well as the human blood index (HBI) in VL endemic villages in India and Nepal. The study had a "before and after intervention" design in 58 households in six clusters. The use of untreated nets reduced the blood feeding rate by 85\% (95\% CI 76.5-91.1\%) and the HBI by 42.2\% (95\% CI 11.1-62.5\%). These results provide circumstantial evidence that untreated nets may provide some degree of personal protection against sand fly bites.

Key words: untreated nets - Phlebotomus argentipes - visceral leishmaniasis - HBI

Visceral leishmaniasis (VL), also known as kalaazar, is an important vector-borne disease in the Indian subcontinent where 150 million people are at risk and more than 40,000 cases are reported annually (WHO 2005). In India, Nepal and Bangladesh, VL is caused by Leishmania donovani (Laveran \& Mesnil) (Kinetoplastida: Trypanosomatidae), which is exclusively transmitted by Phlebotomus argentipes (Annandale \& Brunetti) (Diptera: Psychodidae) (Dinesh et al. 2000).

Control of VL in the India subcontinent is based on detection and treatment of cases and vector control. Indoor residual spraying (IRS) is currently the major form of reducing $P$. argentipes indoor density in the region. IRS was chosen based on the successful control of $\mathrm{VL}$ following the malaria eradication programmes in the 1970s (Ostyn et al. 2008). Despite these efforts, the current strategy of IRS is failing to control VL in the Indian subcontinent. The use of insecticide treated nets (ITN) and, specifically, long-lasting insecticidal nets ( $\mathrm{LN}$ ) have been postulated as alternatives or complements to IRS (Ostyn et al. 2008). The KALANET community trial (ClinicalTrials.gov CT-2005-015374) demonstrated that comprehensive distribution of LN reduces the indoor density of $P$. argentipes by $25 \%$ (Picado et al. 2009).

Other measures including blood feeding rates (percentage of blood fed females) and human blood index $[(\mathrm{HBI})$ the proportion of blood meals taken on man

Financial support: EU-funded INCO-DEV KALANET project (015374)

+ Corresponding author: albert.picado@lshtm.ac.uk

Clive Davies passed away in March 2009.

Received 7 July 2009

Accepted 19 October 2009
(Boreham 1975)] are applied to monitor vector control interventions and can be used to assess personal protection (Githeko et al. 1996, WHO 2006). The use of ITN may enhance the barrier effect of the bed net and/ or reduce the blood feeding rate of those sand flies that cross through the netting, as shown in laboratory bioassays with Phlebotomus perniciosus (Newstead), Phlebotomus papatasi (Scopoli) (Maroli \& Majori 1991) and Lutzomyia longipalpis (Lutz \& Neiva) (Oliveira Filho \& Melo 1994). However, untreated nets may also provide some personal protection against $P$. argentipes based on observational studies on VL in Nepal and Bangladesh (Bern et al. 2000, 2005). Unfortunately, personal protection estimates in VL endemic areas are difficult to generate. This is because human-landing catches, or experimental hut studies, are difficult to justify ethically where the disease is potentially fatal and when, unlike malaria, there is no effective prophylactive to provide to field workers put at risk by such experiments.

The objective of this study was to use the distribution of untreated nets in the households included in the KALANET trial for the night of capture to investigate the impact of untreated nets on the blood feeding rates and anthropophagic behaviour of $P$. argentipes.

Twelve highly endemic clusters for VL included in the KALANET trial were selected in India and Nepal (6 in each country) based on past VL incidence. In September 2006, 10-min aspirations were conducted in 25 randomly selected households per cluster. The 10 households with the highest number of $P$. argentipes in each cluster and their 10 nearest cattle sheds per cluster were monitored from September 2006 for 15 and 16 months in Nepal and India, respectively. LNs were distributed in all the houses in six of the clusters (3 intervention clusters per country) in November and December 2006 in Nepal and India, respectively. The other six clusters were used as controls and did not receive LNs. Monthly 
captures were performed with CDC light traps (LT) set up in the 10 selected houses one night per month from 6 pm-6 am. The following morning between 6-9 am sand flies were collected in the same houses and cattle sheds by aspiration for 15 min each.

From December 2006 in Nepal and from January 2007 in India (1st month that LNs were provided to trial clusters), untreated bed nets were lent to the 10 selected households during the sand fly collection nights in intervention and control clusters. In intervention households LNs were returned on the following morning. The use of untreated nets for the night of capture was required to attribute any observed reduction on P. argentipes indoor density in intervention clusters to the comprehensive distribution of LNs rather than to a local household effect induced by the presence of the LN during the night of capture (Picado et al. 2009).

Informed consent was obtained from the head of the households where sand flies were collected. P. argentipes collected by LT and aspiration were examined under a binocular dissecting microscope for identification of gender and blood meal status. Blood-fed P. argentipes were squashed individually onto Whatman's \#1 filter paper to determine blood meal origin.

An ELISA was used to determine the origin of the blood in fed P. argentipes collected in the 6 KALANET clusters in Nepal. The sand flies were tested for human, bovine, goat, dog, rat and chicken blood. The ELISA protocol used was based on the technique described by Svobodova et al. (2003).

As untreated nets were only provided to households during sand fly collection nights after the first three or four months in Nepal and India, respectively, it was hypothesised that any observed change between pre and post-intervention blood feeding rates and human index in female $P$. argentipes would be best explained by the personal protection provided by the untreated nets.

Since reduction in blood feeding rates in the LN clusters could be due to the personal protection provided by the untreated nets on the night of sand fly capture as well as the presence of LNs throughout the cluster, the effect of untreated nets on blood feeding rates was tested only on control clusters in India and Nepal. However, the effect of untreated nets on human index was assessed using the results from households and cattle sheds from both the intervention and control clusters in Nepal.

The effect of untreated nets was assessed as a "before and after intervention” design using two sets of data: (i) pre and post-intervention LT collections were compared in control households in India and Nepal to determine the effect of untreated nets on the blood feeding rates and (ii) pre and post-intervention collections were compared from intervention and control households and cattle sheds in Nepal to assess the effect of untreated nets on HBI. A negative binomial mixed model (adjusted by country when required) using household or cattle shed as a random effect to control for repeated measures was used. A dichotomous variable representing the time (i.e. pre and post-intervention) was the explanatory variable. In the blood feeding rate and HBI models the number of blood-fed $P$. argentipes and $P$. argentipes with human blood per household (and shed) were the response variables. The interaction term between intervention and time variables was tested to determine whether the effect of the use of untreated nets varied between the two groups.

Ethical clearance to conduct this study was obtained from the Indian Council of Medical Research, the Ethical Committee of the BP Koirala Institute of Health Sciences (Dharan, Nepal) and the corresponding bodies at the In-

TABLE I

Number of female Phlebotomus argentipes collected and percentage of blood fed females by cluster before and after use of untreated nets the night of capture in control clusters in India and Nepal

\begin{tabular}{|c|c|c|c|c|c|c|}
\hline \multirow{2}{*}{$\begin{array}{l}\text { Country } \\
\text { Cluster }\end{array}$} & \multicolumn{3}{|c|}{ Pre-intervention } & \multicolumn{3}{|c|}{ Post-intervention } \\
\hline & LT nights & Females & $\begin{array}{c}\text { Blood fed } \\
\text { (\%) }\end{array}$ & LT nights & Females & $\begin{array}{c}\text { Blood fed } \\
\text { (\%) }\end{array}$ \\
\hline \multicolumn{7}{|l|}{ India } \\
\hline C09 & 32 & 113 & 22 (19.5) & 96 & 106 & $5(4.7)$ \\
\hline C06 & 40 & 85 & $41(48.2)$ & 118 & 112 & $1(0.9)$ \\
\hline C05 & 40 & 83 & $22(26.5)$ & 110 & 121 & $2(1.6)$ \\
\hline Total & 112 & 281 & $85(30.2)$ & 324 & 339 & $8(2.4)$ \\
\hline \multicolumn{7}{|l|}{ Nepal } \\
\hline C60 & 30 & 83 & $7(8.4)$ & 120 & 106 & $1(0.9)$ \\
\hline C56 & 30 & 117 & $16(13.7)$ & 120 & 554 & 19 (3.4) \\
\hline C55 & 30 & 49 & $6(12.2)$ & 120 & 75 & $1(1.3)$ \\
\hline Total & 90 & 249 & 29 (34.3) & 360 & 735 & $21(2.9)$ \\
\hline India and Nepal & 202 & 530 & $114(21.5)$ & 684 & 1074 & $29(2.7)$ \\
\hline
\end{tabular}

the number of nights of capture using CDC light traps (LT nights) per cluster in the three pre- and the 12 post-intervention months is also presented. 


\section{TABLE II}

Number of blood fed females and human blood index (HBI) for Phlebotomus argentipes collected by cluster in households and cattle sheds in Nepal using CDC light traps and aspiration according to the sampling in the three pre and the 12 post-intervention months

\begin{tabular}{|c|c|c|c|c|c|c|c|}
\hline \multirow[b]{2}{*}{ Cluster } & \multirow[b]{2}{*}{ Group } & \multicolumn{3}{|c|}{$\begin{array}{c}\text { Pre-intervention blood fed } \\
\text { P. argentipes }\end{array}$} & \multicolumn{3}{|c|}{$\begin{array}{c}\text { Post-intervention blood fed } \\
\text { P. argentipes }\end{array}$} \\
\hline & & $\begin{array}{l}\text { Household } \\
\text { n (HBI) }\end{array}$ & $\begin{array}{l}\text { Cattle shed } \\
\text { n (HBI) }\end{array}$ & $\begin{array}{c}\text { Total } \\
\mathrm{n}(\mathrm{HBI})\end{array}$ & $\begin{array}{l}\text { Household } \\
\mathrm{n}(\mathrm{HBI})\end{array}$ & $\begin{array}{l}\text { Cattle shed } \\
\text { n (HBI) }\end{array}$ & $\begin{array}{c}\text { Total } \\
\mathrm{n}(\mathrm{HBI})\end{array}$ \\
\hline C51 & intervention & $28(75)$ & $3(67)$ & 31 (74) & $4(0)$ & $25(4)$ & $29(3)$ \\
\hline C52 & intervention & $12(92)$ & $0(0)$ & 12 (92) & $4(50)$ & $0(0)$ & $4(50)$ \\
\hline C53 & intervention & $4(100)$ & $1(100)$ & $5(100)$ & $4(100)$ & $0(0)$ & $4(100)$ \\
\hline Total intervention & & $44(82)$ & $4(75)$ & 48 (81) & $12(50)$ & $25(4)$ & 37 (19) \\
\hline C55 & control & $4(50)$ & $0(0)$ & $4(50)$ & $1(0)$ & $1(0)$ & $2(0)$ \\
\hline C56 & control & 12 (83) & $0(0)$ & 12 (83) & 38 (87) & $15(27)$ & $53(70)$ \\
\hline C60 & control & $8(88)$ & $0(0)$ & $8(88)$ & $4(50)$ & $0(0)$ & $4(50)$ \\
\hline Total control & & 24 (79) & $0(0)$ & 24 (79) & 43 (81) & $16(25)$ & $59(66)$ \\
\hline Total intervention and control & & $68(81)$ & $4(75)$ & $72(81)$ & $55(75)$ & $41(12)$ & $96(48)$ \\
\hline
\end{tabular}

stitute of Tropical Medicine (Antwerp, Belgium) and the London School of Hygiene and Tropical Medicine (UK).

A total of 1,064 female $P$. argentipes were collected using LTs from 58 households in the six KALANET control clusters. Of these, 143 (11\%) were blood-fed. As shown in Table I, a large post-intervention reduction in blood-fed rates was observed in all six control clusters (from 21.5 to $2.7 \%$ ). The negative binomial mixed model demonstrates that the percentage of female $P$. argentipes with blood meals dropped significantly by $85.5 \%$ (95\% CI $76.5-91.1 \%$, p $<0.001)$ after the untreated nets were introduced.

In order to determine the human blood index, the 168 blood-fed P. argentipes collected in Nepal using LT and aspiration in households and cattle sheds over 15 months were analysed for blood origin. Of these samples, $61.9 \%$ fed on human blood, $22.6 \%$ on bovine, $4.2 \%$ on dog, $3 \%$ on goat and $0.6 \%$ on chicken. We were unable to identify the blood origin for $10.1 \%$ of the samples. A total of four samples had mixed blood meals: two human/bovine, one human/goat and one human/chicken mixtures were found. These results show that $P$. argentipes in VL endemic foci in Terai are mainly anthropophilic but will feed on bovine blood meal when available.

To evaluate the effect of untreated nets on HBI in Nepal, the ELISA results summarised in Table II were dichotomised (i.e. human blood vs. non-human blood). Samples not identified were considered as non-human blood in the analyses to avoid overestimations of the effect of untreated nets. The negative binomial mixed model found that HBI was reduced significantly by 42.2\% (95\% CI 11.1-62.5\%, $\mathrm{p}=0.014$ ) when untreated nets were used. The overall reduction in the control and intervention clusters was significantly different (interaction term: $\mathrm{p}=0.014$ ). This result suggests that the massive distribution of LNs in intervention clusters had an effect on the blood feeding behaviour of $P$. argentipes, as HBI was reduced to a greater extent in intervention compared to control clusters (Table II). This phenomena has been described in laboratory reared sand flies (Maroli \& Majori 1991, Oliveira Filho \& Melo 1994) and field trials with mosquitoes in India (Sampath et al. 1998, Dinesh et al. 2000), but it could not be tested (by comparing post-intervention HBI between intervention and control clusters) in this trial due to the massive reduction of blood-fed $P$. argentipes in households post-intervention.

These results provide circumstantial evidence that even untreated nets provide personal protection against sand fly bites. Blood-fed rates in LT catches decreased by $85.5 \%$ in houses using untreated nets. Further, HBI was reduced by $42.2 \%$ suggesting that female P. argentipes are diverted from humans when untreated nets are used (the later effect seems to be enhanced by the use of $\mathrm{LNs}$ ). This result is consistent with observational studies that have detected a significant negative association between the ownership of untreated bed nets and the incidence of VL in endemic areas in Nepal and Bangladesh (Bern et al. 2000, 2005); however, other studies in Nepal have failed to detect such an effect (Schenkel et al. 2006). Since there were no concurrent controls, the reduction in blood feeding rate and HBI could have been due to another confounding time-associated factor in our study. For example: (i) meteorological factors that may alter the frequency of vector-host contact, e.g., changes in temperature or humidity and (ii) changes in host availability, e.g., more domestic animals may have been available as alternative hosts during the course of the trial. Specific entomological trials should be conducted to corroborate the results observed in this study.

\section{REFERENCES}

Bern C, Hightower AW, Chowdhury R, Ali M, Amann J, Wagatsuma Y, Haque R, Kurkjian K, Vaz LE, Begum M, Akter T, CetreSossah CB, Ahluwalia IB, Dotson E, Secor WE, Breiman RF, Maguire JH 2005. Risk factors for kala-azar in Bangladesh. Emerg Infect Dis 11: 655-662. 
Bern C, Joshi AB, Jha SN, Das ML, Hightower A, Thakur GD, Bista MB 2000. Factors associated with visceral leishmaniasis in Nepal: bed-net use is strongly protective. Am J Trop Med Hyg 63: 184-188.

Boreham PF 1975. Some applications of bloodmeal identifications in relation to the epidemiology of vector-borne tropical diseases. J Trop Med Hyg 78: 83-91.

Dinesh DS, Kar SK, Kishore K, Palit A, Verma N, Gupta AK, Chauhan DS, Singh D, Sharma VD, Katoch VM 2000. Screening sand flies for natural infection with Leishmania donovani, using a non-radioactive probe based on the total DNA of the parasite. Ann Trop Med Parasitol 94: 447-451.

Githeko AK, Mbogo CMM, Curtis CF, Lines J, Lengeler C 1996. Entomological monitoring of large-scale vector-control interventions. Parasitol Today 12: 127-128.

Maroli M, Majori G 1991. Permethrin-impregnated curtains against phlebotomine sand flies (Diptera: Psychodidae): laboratory and field studies. Parassitologia 33 (Suppl.): 399-404.

Oliveira Filho AM, Melo MT 1994. Vectors control importance on leishmaniasis transmission. Mem Inst Oswaldo Cruz 89: 451-456.

Ostyn B, Vanlerberghe V, Picado A, Dinesh DS, Sundar S, Chappuis F, Rijal S, Dujardin JC, Coosemans M, Boelaert M, Davies C 2008. Vector control by insecticide-treated nets in the fight against visceral leishmaniasis in the Indian subcontinent, what is the evidence? Trop Med Int Health 13: 1073-1085.
Picado A, Das ML, Kumar V, Kesari S, Dinesh DS, Roy L, Rijal S, Das P, Rowland M, Sundar S, Coosemans M, Boelaert M, Davies CR 2009. Effect of village-wide use of long lasting insecticidal nets on visceral leishmaniasis vectors in India and Nepal: clusterrandomized trial. PLoS Negl Trop Dis, in press.

Sampath TR, Yadav RS, Sharma VP, Adak T 1998. Evaluation of lambdacyhalothrin-impregnated bednets in a malaria endemic area of India. Part 2. Impact on malaria vectors. J Am Mosq Control Assoc 14: 437-443.

Schenkel K, Rijal S, Koirala S, Koirala S, Vanlerberghe V, Van der Stuyft P, Gramiccia M, Boelaert M 2006. Visceral leishmaniasis in Southeastern Nepal: a cross-sectional survey on Leishmania donovani infection and its risk factors. Trop Med Int Health 11: 1792-1799.

Svobodová M, Sádlová J, Chang KP, Volf P 2003. Short report: distribution and feeding preference of the sand flies Phlebotomus sergenti and $P$. papatasi in a cutaneous leishmaniasis focus in Sanliurfa, Turkey. Am J Trop Med Hyg 68: 6-9.

WHO - World Health Organisation 2005. Regional Technical Advisory Group on kala-azar elimination. Report of the first meeting, Manesar, Haryana, 20-23 December 2004, Document SEAVBC-88, New Delhi. Avaliable from: http://www.serao.who.int/ LinFiles/Kala_azar_VBC-88.pdf.

WHO - World Health Organisation 2006. Guidelines for testing mosquito adulticides for indoor residual spraying and treatment of mosquito nets, Document WHO/CDS/NTD/WHOPES/GCDPP/2006.3, Geneva. Avaliable from: http://whqlibdoc.who.int/ HQ/2006/WHO_CDS_NTD_WHOPES_GCDPP_2006.3_eng.pdf. 